\title{
Status Review of Theory Predictions for Charged Higgs Cross Sections and Branching Ratios
}

\author{
Sven Heinemeyer* \\ Instituto de Física de Cantabria (CSIC-UC), Santander, Spain \\ E-mail: Sven.Heinemeyer@cern.ch
}

In order to identify the Higgs sector realized in nature, the predictions for Higgs boson masses, production cross sections and decay widths have to be compared with experimental results. We give a brief overview about the status of the evaluation of the properties of charged Higgs bosons, mostly focusing on the case of the Minimal Supersymmetric Standard Model (MSSM).

Prospects for Charged Higgs Discovery at Colliders

October 8-11, 2012

Uppsala University Sweden

*Speaker. 


\section{Introduction}

Identifying the mechanism of electroweak symmetry breaking is one of the main goals of the LHC. The spectacular discovery of a Higgs-like particle with a mass around $M_{H} \simeq 126 \mathrm{GeV}$, which has been announced by ATLAS [1] and CMS [2], marks a milestone of an effort that has been ongoing for almost half a century and opens up a new era of particle physics. However, even after accepting that this new particle is $a$ Higgs boson, the crucial question remains what is the underlying model, how exactly is electroweak symmetry breaking realized. Many possibilities have been studied in the past, of which the most popular ones are the Higgs mechanism within the Standard Model (SM) [3], the Two Higgs Doublet Model (THDM) [4], and within supersymmetric (SUSY) models [5], which naturally contain at least two Higgs doublets. While the SM contains only one physical (neutral) Higgs boson, models with two Higgs doublets contain, besides three neutral Higgs bosons, at least two charged Higgs bosons, $H^{ \pm}$. Any evidence for a charged Higgs boson would be an unambiguous sign of an extended Higgs sector, i.e. of physics beyond the SM. However, even in the case of a charged Higgs discovery, the true nature of this object has to be further determined, as it could be a charged Higgs boson in the THDM, the MSSM or even more extended models such as the NMSSM.

In order to identify the Higgs sector realized in nature, the predictions for couplings to SM fermions and bosons as well as self-couplings have to be compared with the experimental results. To perform this task precise theory predictions are needed for Higgs production cross sections and decay widths (or at best for complete processes). Here we give a brief overview about this kind of calculations concerning the charged Higgs bosons.

Within the THDM the Higgs potential contains 14 independent parameters, and the mass of the charged Higgs boson, $M_{H^{ \pm}}$, is usually treated as a free parameter. A dedicated code for production cross sections and branching ratios in the THDM has been published in Ref. [6].

Within SUSY many results for the charged Higgs boson beyond the tree-level are available in the Minimal Supersymmetric Standard Model (MSSM), see below. The only code that contains higher-order corrections for a charged Higgs boson beyond the MSSM is NMSSMTools [7], providing masses, production cross sections and decay widths in the NMSSM, i.e. the MSSM with an additional Higgs singlet. In the following we will focus on the MSSM.

\section{The charged Higgs in the MSSM}

The Higgs sector of the MSSM contains two Higgs doublets, leading to five physical Higgs bosons. At tree-level these are the light and heavy $\mathscr{C} \mathscr{P}$-even $h$ and $H$, the $\mathscr{C} \mathscr{P}$-odd $A$ and the charged $H^{ \pm}$. At lowest order the Higgs sector can be described besides the SM parameters by two additional independent parameters, chosen to be the mass of the $A$ boson, $M_{A}$ (in the case of vanishing complex phases), and $\tan \beta \equiv v_{2} / v_{1}$, the ratio of the two vacuum expectation values. Accordingly, all other masses and couplings can be predicted at tree-level, e.g. the charged Higgs boson mass

$$
m_{H^{ \pm}}^{2}=M_{A}^{2}+M_{W}^{2}
$$


$M_{Z, W}$ denote the masses of the $Z$ and $W$ boson, respectively. This tree-level relation receives higher-order corrections, where the loop corrected charged Higgs-boson mass is denoted as $M_{H^{ \pm}}$. Three codes exist for the calculation of $M_{H^{ \pm}}$and the various decay widths, FeynHiggs [8-12], CPsuperH [13] and Hdecay [14].

The charged Higgs bosons of the MSSM (or a more general Two Higgs Doublet Model) have been searched at LEP, the Tevatron and the LHC, and will be searched for (or hopefully analyzed at) a Linear Collider such as ILC or CLIC. The LEP searches $[15,16]$ yielded a robust bound of $M_{H^{ \pm}} \gtrsim 80 \mathrm{GeV}$ [17]. The Tevatron bounds [18] are by now superseeded by the LHC charged Higgs searches [19]. Those searches are especially sensitive at lower $M_{A}$ and large $\tan \beta$ or very low $\tan \beta$, extending the LEP bounds in this region of parameter space. At the LHC the prospects for charged Higgs boson searches are best at large $\tan \beta$, reaching up to $M_{A} \lesssim 800 \mathrm{GeV}$. At the ILC, if the charged Higgs is in the kinematical reach, a high-precision determination of the charged Higgs boson properties will be possible [20,21].

The main production channels at the LHC are

$$
\begin{array}{r}
p p \rightarrow t \bar{t}+X, \quad t \bar{t} \rightarrow t H^{-} \bar{b} \text { or } H^{+} b \bar{t}, \\
g b \rightarrow H^{-} t \text { or } g \bar{b} \rightarrow H^{+} \bar{t} .
\end{array}
$$

The decay used in the analysis to detect the charged Higgs boson is

$$
H^{ \pm} \rightarrow \tau v_{\tau} \rightarrow \text { hadrons } v_{\tau}
$$

The "light charged Higgs boson" is characterized by $M_{H^{ \pm}}<m_{t}$. The main production channel is given in Eq. (2.2). Close to threshold also Eq. (2.3) contributes. The relevant (i.e. detectable) decay channel is given by Eq. (2.4).

The "heavy charged Higgs boson" is characterized by $M_{H^{ \pm}} \gtrsim m_{t}$. Here Eq. (2.3) gives the largest contribution to the production cross section, and very close to threshold Eq. (2.2) can contribute somewhat. The relevant decay channel is again given in Eq. (2.4).

\section{Production cross section of the light charged Higgs}

To estimate the cross section for events with charged Higgs bosons in top quark pair production, the following ingredients are needed: The top quark pair production cross section, the branching ratio $\mathrm{BR}\left(t \rightarrow b H^{+}\right)$and the light charged Higgs boson decay branching ratios (more details can be found in Ref. [22]). In the following we will concentrate on the $7 \mathrm{TeV}$ case, while the $8 \mathrm{TeV}$ case can be analyzed analogously.

Top quark pair production cross section:

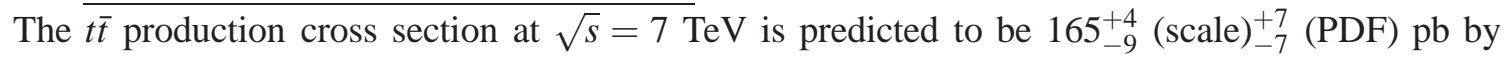
approximate NNLO calculations [23,24] recommended by the ATLAS Top Working Group [25]. The scale uncertainty is obtained as the "envelope" from a variation of the renormalization scale $\mu_{R}$ and the factorization scale $\mu_{\mathrm{F}}$ from 0.5 to 2 times $m_{t}$ (with $0.5 \leq \mu_{\mathrm{F}} / \mu_{\mathrm{R}} \leq 2$ ). The PDF uncertainty, obtained using MSTW2008 [26], is taken at the 68\% C.L.; both uncertainties should be added linearly. Finally, the uncertainty in the production cross section from the experimental uncertainty 
in $m_{t}$ is estimated to be

$$
\Delta \sigma / \sigma \sim 5 \Delta m_{t}^{\exp } / m_{t}
$$

This uncertainty is combined quadratically with the scale/PDF uncertainty. However, the contribution to the overall uncertainty is relatively small.

Top quark decays via a charged Higgs boson:

The decay width calculation of the top quark to a light charged Higgs boson is compared for two different programs, FeynHiggs and Hdecay, see above.

The FeynHiggs calculation is based on the evaluation of $\Gamma\left(t \rightarrow W^{+} b\right)$ and $\Gamma\left(t \rightarrow H^{+} b\right)$. The former is calculated at NLO according to Ref. [27]. The decay to the charged Higgs boson and the bottom quark uses $m_{t}\left(m_{t}\right)$ and $m_{b}\left(m_{t}\right)$ in the Yukawa coupling, where the latter receives the additional correction factor $1 /\left(1+\Delta_{b}\right)$. The numerical results reviewed here are based on the evaluation of $\Delta_{b}$ in Ref. [28]. Furthermore additional QCD corrections taken from Ref. [29] are included.

The Hdecay calculation is based on the evaluation of $\Gamma\left(t \rightarrow W^{+} b\right)$ and $\Gamma\left(t \rightarrow H^{+} b\right)$. The decays were evaluated including the full NLO QCD corrections (including bottom mass effects) [27] (and references therein). The top and (kinematical) bottom masses are taken as the pole masses while the bottom mass of the Yukawa coupling is taken as running $\overline{\mathrm{MS}}$ mass at the scale of the top mass. SUSY QCD and electroweak corrections are approximated via $\Delta_{b}$ based on Refs. [29-32].

In Fig. 1 we show the comparison between the evaluations of the decay $t \rightarrow H^{+} b$ in FeynHiggs and Hdecay [22]. The top row shows the decay width, while the bottom row contains the result for the branching ratios. The parameters are chosen according to the $m_{h}^{\max }$ scenario [33] with $\mu$ set to 200 (1000) GeV in the left (right) column. One can see that the agreement between the two codes, despite the differences in the $\Delta_{b}$ evaluation is excellent. In the following we assume a $\sim 6 \%$ uncertainty in the top decays to missing one-loop electroweak and two-loop QCD corrections.

Light charged Higgs boson decay:

Within the $m_{h}^{\max }$ scenario of the $\operatorname{MSS} M$, the $\operatorname{BR}\left(H^{ \pm} \rightarrow \tau v_{\tau}\right) \approx 1$ for all parameter values still allowed by the LEP experiments [17]. (Only for very large values of $M_{H^{ \pm}}$the off-shell decay to $t b$ can reach a level of up to $10 \%$. The strong dominance of $H^{ \pm} \rightarrow \tau \nu_{\tau}$ holds for most of the MSSM parameter space if not $\tan \beta$ becomes very small.) The uncertainty on this assumption is less than $1 \%$ and thus negligible compared to other uncertainties.

The overall uncertainty, evaluated in the $m_{h}^{\max }$ scenario can be seen in Fig. 2. We show

$$
\sigma_{t t} \cdot \mathrm{BR}\left(t \rightarrow b H^{ \pm}\right) \cdot \mathrm{BR}\left(t \rightarrow b W^{ \pm}\right) \cdot 2
$$

for $\sqrt{s}=7 \mathrm{TeV}$ as a function of $M_{H^{ \pm}}$with all the above uncertainties combined. The result is shown for $\tan \beta=5,10,30,50$. As can be seen, the uncertainties are still substantial. They have to be taken into account for reliable and robust bounds on the MSSM parameter space from the nonobservation of a light charged Higgs. Conversely, using a potential observation of a light charged Higgs for a determination of the underlying parameters would require a substantial reduction of the uncertainties. 

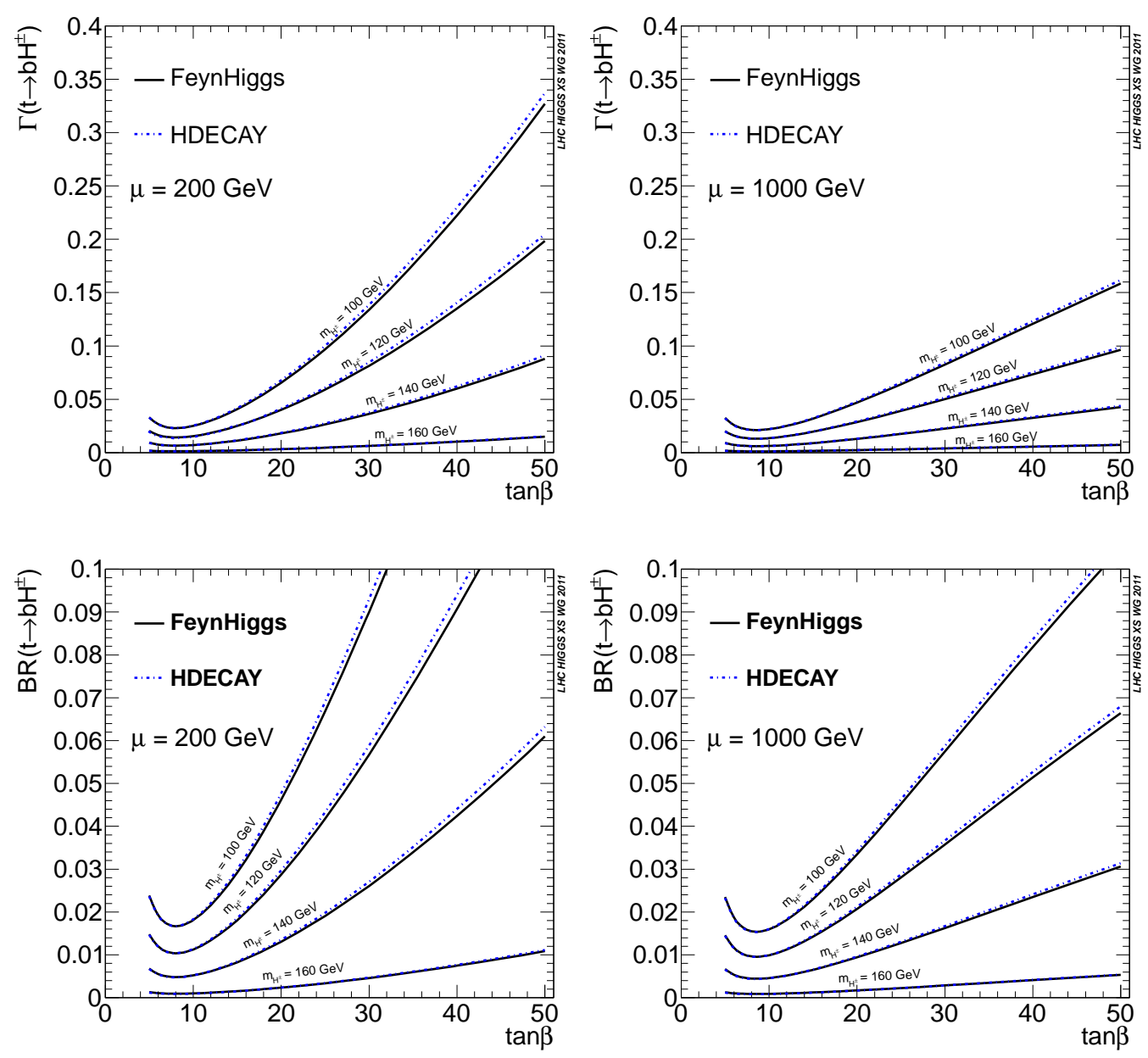

Figure 1: Comparison of the $\Gamma\left(t \rightarrow H^{+} b\right)$ (upper row) and $\mathrm{BR}\left(t \rightarrow H^{+} b\right)$ (lower row) between FeynHiggs and Hdecay. The results are shown for various values of $M_{H^{ \pm}}$and for $\mu=200$ (1000) GeV in the left (right) column.

\section{Production cross section of the heavy charged Higgs}

For heavy charged Higgs bosons, $M_{H^{ \pm}} \gtrsim m_{t}$, associated production $p p \rightarrow t b H^{ \pm}+\mathrm{X}$ is the dominant production mode. Two different formalisms can be employed to calculate the cross section for associated $t b H^{ \pm}$production. In the four-flavor scheme (4FS) with no $b$ quarks in the initial state, the lowest-order QCD production processes are gluon-gluon fusion and quark-antiquark annihilation, $g g \rightarrow t b H^{ \pm}$and $q \bar{q} \rightarrow t b H^{ \pm}$, respectively.

On the other hand, potentially large logarithms $\propto \ln \left(\mu_{\mathrm{F}} / m_{b}\right)$, which arise from the splitting of incoming gluons into nearly collinear $b \bar{b}$ pairs, can be summed to all orders in perturbation theory by introducing bottom parton densities, i.e. in the five flavor scheme (5FS) [34]. To all orders in perturbation theory the four- and five-flavor schemes are identical, but the way of ordering the perturbative expansion is different, and the results do not match exactly at finite order. For more details see Ref. [22] and references therein. 


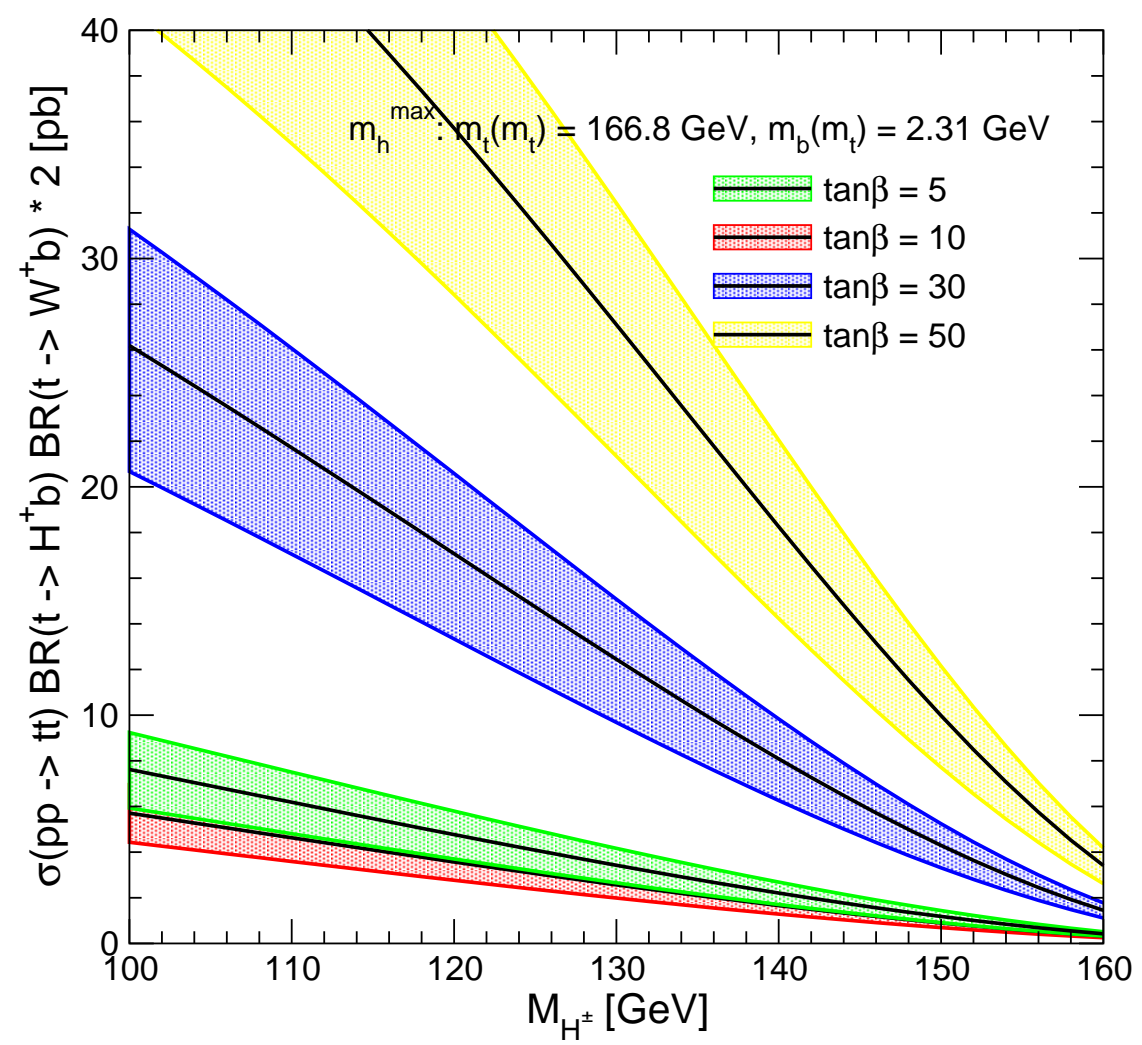

Figure 2: $\sigma_{t t} \cdot \mathrm{BR}\left(t \rightarrow b H^{ \pm}\right) \cdot \mathrm{BR}\left(t \rightarrow b W^{ \pm}\right) \cdot 2$ including scale and PDF uncertainties, uncertainties for missing electroweak and QCD corrections, and $\Delta_{b}$-induced uncertainties for $\sqrt{s}=7 \mathrm{TeV}$.

Santander matching:

A simple and pragmatic formula for the combination of the four- and five-flavor scheme calculations of bottom-quark associated Higgs-boson production has been suggested in Ref. [35], the so-called "Santander matching". The main idea behind this matching scheme is the following: The 4FS and 5FS calculations provide the unique description of the cross section in the asymptotic limits $M_{H} / m_{b} \rightarrow 1$ and $M_{H} / m_{b} \rightarrow \infty$, respectively (where $M_{H}$ denotes a generic Higgs boson mass, i.e. the arguments are valid for the neutral as well as for the charged Higgs production). The two approaches are combined in such a way that they are given a weight, depending on the value of the Higgs-boson mass. Since the difference between the 4FS and the 5FS is logarithmic, the dependence of their relative importance on $M_{H}$ should be controlled by a logarithmic term. Consequently, the proposal for the "Santander matching" reads [35],

$$
\sigma^{\text {matched }}=\frac{\sigma^{4 \mathrm{FS}}+t \sigma^{5 \mathrm{FS}}}{1+t}
$$

with the weight $w$ defined as

$$
t=\ln \frac{M_{H}}{m_{b}}-2,
$$

and $\sigma^{4 \mathrm{FS}}$ and $\sigma^{5 \mathrm{FS}}$ denote the total inclusive cross section in the 4FS and the 5FS, respectively. The theoretical uncertainties in the 4FS and the 5FS calculations should be added linearly, using 
the weights $t$. In this way it is ensured that the combined error is always larger than the minimum of the two individual errors [35]:

$$
\Delta \sigma_{ \pm}=\frac{\Delta \sigma_{ \pm}^{4 \mathrm{FS}}+t \Delta \sigma_{ \pm}^{5 \mathrm{FS}}}{1+t}
$$

where $\Delta \sigma_{ \pm}^{4 \mathrm{FS}}$ and $\Delta \sigma_{ \pm}^{5 \mathrm{FS}}$ denote the upper/lower uncertainty limits of the 4FS and the 5FS, respectively.

Numerical results are revied in Fig. 3. In the left plot we show the central values for the total inclusive cross section in the 5FS (red, dashed), the 4FS (green, dashed), and for the matched cross section (blue, solid). They have been evaluated as a function of $M_{H^{ \pm}}$for $\sqrt{s}=7 \mathrm{TeV}, \tan \beta=30$, all SUSY corrections omitted and using the MSTW2008 PDF set [26]. The right plot shows the corresponding uncertainty bands with the same color coding. An updated approach with a new choice of the renormalization scale [36] has recently been presented [37], but still requires further studies.
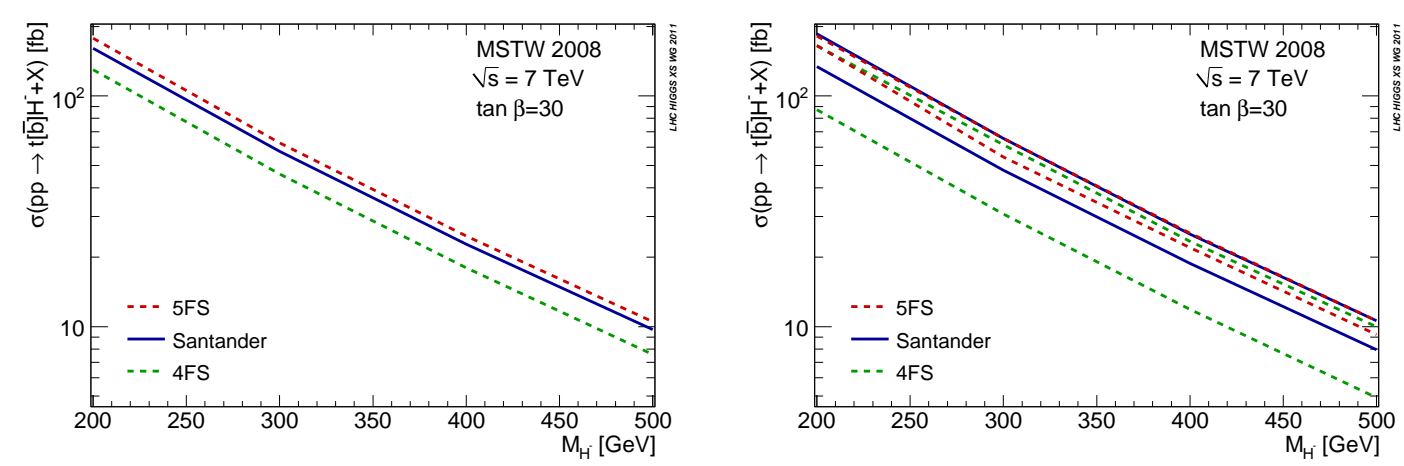

Figure 3: Left: central values for the total inclusive cross section in the 5FS (red, dashed), the 4FS (green, dashed), and for the matched cross section (blue, solid) as a function of $M_{H^{ \pm}}$for $\sqrt{s}=7 \mathrm{TeV}, \tan \beta=30$, all SUSY corrections omitted. Right: corresponding theory uncertainty bands for the total inclusive cross section with the same color coding.

\section{Charged Higgs branching ratios}

An analysis for the charged Higgs branching ratios so far only exists for the light charged Higgs case. The charged Higgs boson decay widths are calculated with FeynHiggs and Hdecay in the $m_{h}^{\max }$ benchmark scenario, where details can be found in Ref. [22]. The decay channels $H^{ \pm} \rightarrow \tau v_{\tau}, H^{ \pm} \rightarrow A W^{ \pm}, H^{ \pm} \rightarrow c s, H^{ \pm} \rightarrow H W^{ \pm}, H^{ \pm} \rightarrow \mu v_{\mu}$ and $H^{ \pm} \rightarrow t b$ available in both programs are studied, where the latter is mostly negligible for the light charged Higgs. For $H^{ \pm} \rightarrow$ $\tau \nu_{\tau}$, FeynHiggs includes the Higgs propagator corrections up to the two-loop level. Concerning the latter, in Hdecay these corrections are included in the approximation of vanishing external momentum. On the other hand, it includes the full NLO QCD corrections to charged Higgs decays into quarks, which are incorporated in FeynHiggs only in the approximation of a heavy charged Higgs boson. 
The experimentally most interesting decay channel $H^{ \pm} \rightarrow \tau v_{\tau}$ shows a good agreement, as can be seen in the upper row in Fig. 4 [22]. Here Hdecay consistently predicting a 3.5\% larger decay width than FeynHiggs, due to the differences described above. A good agreement is also found in the $H^{ \pm} \rightarrow \mu v_{\mu}$ channel (not shown), again with Hdecay predicting consistently a $\sim 3.5 \%$ larger decay width than FeynHiggs. In the $H^{ \pm} \rightarrow c s$ channel, presented in the lower row of Fig. 4 [22], a notable discrepancy of 7-19\% is found. The differences in this channel may be attributed to the QCD corrections implemented in FeynHiggs, which are valid only in the limit of heavy charged Higgs masses (in comparison to the quark masses), whereas in Hdecay they are more complete. Conversely, the evaluation of $\Delta_{s}$, which is crucial for the calculation of $\Gamma\left(H^{ \pm} \rightarrow c s\right)$, is more complete in FeynHiggs. This channel can only play a significant role for very low values of $\tan \beta$, and is numerically negligible within the $m_{h}^{\max }$ scenario.
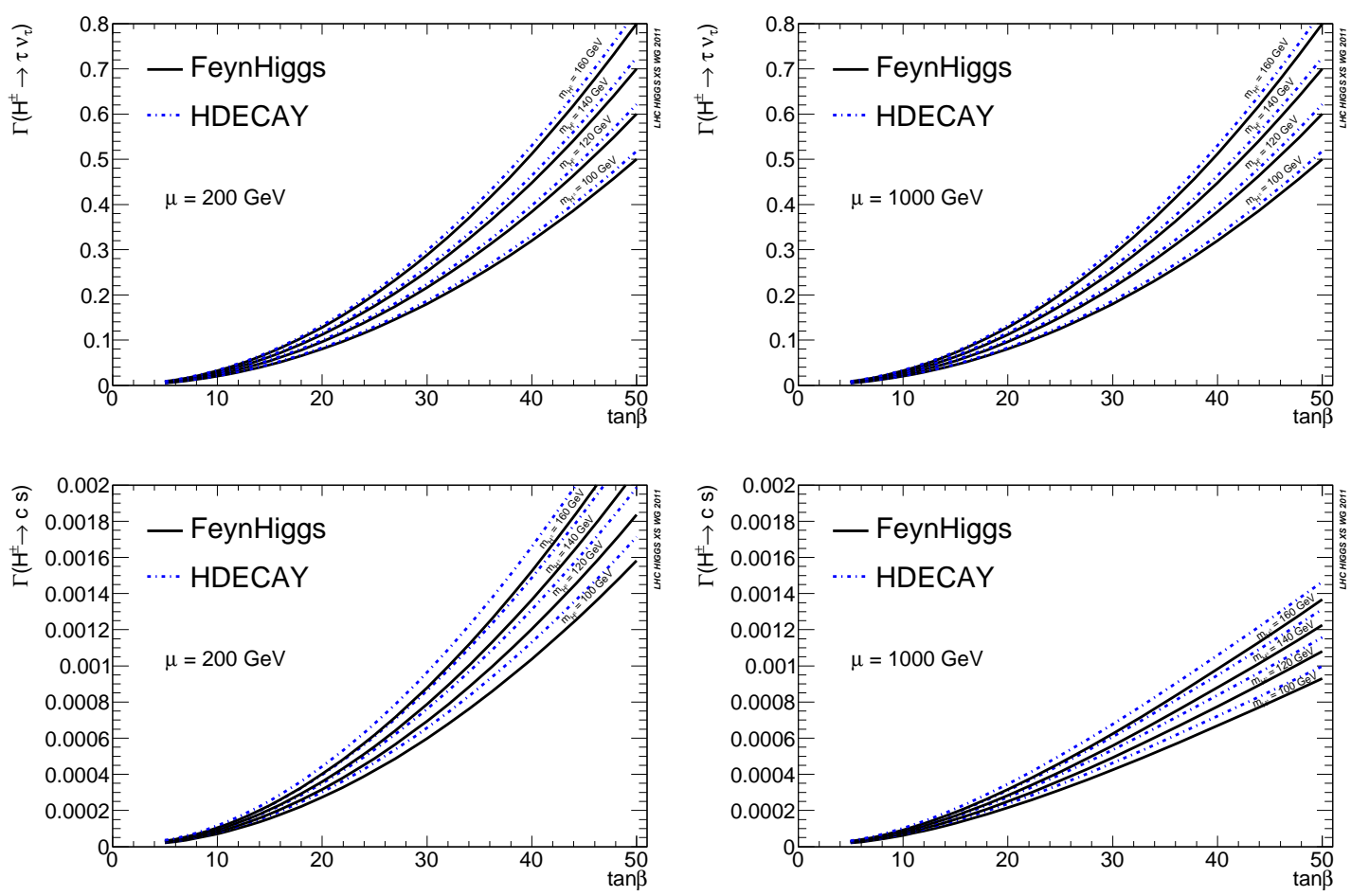

Figure 4: Comparison of the $\Gamma\left(H^{ \pm} \rightarrow \tau v_{\tau}\right)$ (upper row) and $\Gamma\left(H^{ \pm} \rightarrow c s\right)$ (lower row) between FeynHiggs and Hdecay in the $m_{h}^{\max }$ scenario. The results are shown for various values of $M_{H^{ \pm}}$and for $\mu=$ 200(1000) GeV in the left (right) column [22].

\section{Conclusions}

The current status of cross section and branching ratio calculation for charged Higgs bosons in the MSSM has been reviewed. For the light charged Higgs boson $\left(M_{H^{ \pm}}<m_{t}\right)$ the uncertainties have been evaluated in the $m_{h}^{\max }$ scenario and are still substantial. The production cross section for a heavy charged Higgs $\left(M_{H^{ \pm}}>m_{t}\right)$ are evaluated using the "Santander matching" scheme, combining the 4FS and the 5FS. An analysis for the charged Higgs branching ratios is available 
only for a light charged Higgs boson. A comparison between FeynHiggs and Hdecay shows good agreement for the leptonic channels but a $\mathscr{O}(10 \%)$ difference in the (subdominant) $H^{ \pm} \rightarrow c s$ decay channel.

Overall, a reliable and robust derivation of bounds on the MSSM parameter space from the non-observation of a charged Higgs, as well as a determination of the underlying parameters from a potential observation of a charged Higgs will require a substantial reduction of the present uncertainties.

\section{Acknowledgements}

The work of S.H. was partially supported by CICYT (grant FPA 2010-22163-C02-01) and by the Spanish MICINN's Consolider-Ingenio 2010 Programme under grant MultiDark CSD200900064.

\section{References}

[1] G. Aad et al. [ATLAS Collaboration], Phys. Lett. B 716 (2012) 1 [arXiv:1207.7214 [hep-ex]].

[2] S. Chatrchyan et al. [CMS Collaboration], Phys. Lett. B 716 (2012) 30 [arXiv:1207.7235 [hep-ex]].

[3] S. Glashow, Nucl. Phys. 22 (1961) 579; S. Weinberg, Phys. Rev. Lett. 19 (1967) 19; A. Salam, in: Proceedings of the 8th Nobel Symposium, Editor N. Svartholm, Stockholm, 1968.

[4] S. Weinberg, Phys. Rev. Lett. 37 (1976) 657; J. Gunion, H. Haber, G. Kane and S. Dawson, The Higgs Hunter's Guide (Perseus Publishing, Cambridge, MA, 1990), and references therein.

[5] H. Nilles, Phys. Rept. 110 (1984) 1; H. Haber and G. Kane, Phys. Rept. 117 (1985) 75; R. Barbieri, Riv. Nuovo Cim. 11 (1988) 1.

[6] D. Eriksson, J. Rathsman and O. Stål, Comput. Phys. Commun. 181 (2010) 189 [arXiv:0902.0851 [hep-ph]]; Comput. Phys. Commun. 181 (2010) 833.

[7] U. Ellwanger and C. Hugonie, Comput. Phys. Commun. 177 (2007) 399 [arXiv:hep-ph/0612134]; Comput. Phys. Commun. 175 (2006) 290 [arXiv:hep-ph/0508022]. U. Ellwanger, J. Gunion and C. Hugonie, JHEP 0502 (2005) 066 [arXiv:hep-ph/0406215].

[8] S. Heinemeyer, W. Hollik and G. Weiglein, Comput. Phys. Commun. 124 (2000) 76, [arXiv:hep-ph/9812320]; T. Hahn, S. Heinemeyer, W. Hollik, H. Rzehak and G. Weiglein, Comput. Phys. Commun. 180 (2009) 1426; see: www . feynhiggs . de .

[9] S. Heinemeyer, W. Hollik and G. Weiglein, Eur. Phys. J. C 9 (1999) 343 [arXiv:hep-ph/9812472].

[10] G. Degrassi, S. Heinemeyer, W. Hollik, P. Slavich and G. Weiglein, Eur. Phys. J. C 28 (2003) 133 [arXiv:hep-ph/0212020].

[11] M. Frank, T. Hahn, S. Heinemeyer, W. Hollik, H. Rzehak and G. Weiglein, JHEP 0702 (2007) 047 [arXiv:hep-ph/0611326].

[12] S. Heinemeyer, W. Hollik, H. Rzehak and G. Weiglein, Phys. Lett. B 652 (2007) 300 [arXiv:0705.0746 [hep-ph]].

[13] J. Lee, A. Pilaftsis et al., Comput. Phys. Commun. 156 (2004) 283 [arXiv:hep-ph/0307377]; J. Lee, M. Carena, J. Ellis, A. Pilaftsis and C. Wagner, Comput. Phys. Commun. 180 (2009) 312 [arXiv:0712.2360 [hep-ph]]; arXiv:1208.2212 [hep-ph]. 
[14] A. Djouadi, J. Kalinowski and M. Spira, Comput. Phys. Commun. 108 (1998) 56 [arXiv:hep-ph/9704448].

[15] A. Heister et al. [ALEPH Collaboration], Phys. Lett. B 543 (2002) 1 [arXiv:hep-ex/0207054]; J. Abdallah et al. [DELPHI Collaboration], Eur. Phys. J. C 34 (2004) 399 [arXiv:hep-ex/0404012]; P. Achard et al. [L3 Collaboration], Phys. Lett. B 575 (2003) 208 [arXiv:hep-ex/0309056]; D. Horvath [OPAL Collaboration], Nucl. Phys. A 721 (2003) 453.

[16] [LEP Higgs working group], arXiv:hep-ex/0107031.

[17] P. Lutz et al. [LEP Higgs working group], in preparation, see also: ilcagenda.linearcollider.org/ contributiondisplay $\cdot$ py? contribId=153\&session Id=71\& conf $I d=1296$.

[18] T. Aaltonen et al. [CDF Collaboration], Phys. Rev. Lett. 103 (2009) 101803 [arXiv:0907.1269 [hep-ex]]; V. Abazov et al. [DØCollaboration], Phys. Lett. B 682 (2009) 278 [arXiv:0908.1811 [hep-ex]]; P. Gutierrez [CDF and DØCollaborations], PoS CHARGED 2010 (2010) 004.

[19] G. Aad et al. [ATLAS Collaboration], JHEP 1206 (2012) 039 [arXiv:1204.2760 [hep-ex]]; S. Chatrchyan et al. [CMS Collaboration], JHEP 1207 (2012) 143 [arXiv:1205.5736 [hep-ex]].

[20] S. Heinemeyer et al., arXiv:hep-ph/0511332.

[21] A. Ferrari, talk given at the CHärged 2006, Uppsala, Sweden, September 2006.

[22] S. Dittmaier et al., arXiv:1201.3084 [hep-ph].

[23] S. Moch and P. Uwer, Nucl. Phys. Proc. Suppl. 183 (2008) 75 [arXiv:0807.2794 [hep-ph]].

[24] U. Langenfeld, S. Moch and P. Uwer, arXiv:0907.2527 [hep-ph].

[25] G. Aad et al. [ATLAS Collaboration], Phys. Lett. B 707 (2012) 459 [arXiv:1108.3699 [hep-ex]].

[26] A. Martin, W. Stirling, R. Thorne and G. Watt, Eur. Phys. J. C 63 (2009) 189 [arXiv:0901.0002 [hep-ph]].

[27] J. Campbell, R. Ellis and F. Tramontano, Phys. Rev. D 70 (2004) 094012 [hep-ph/0408158].

[28] L. Hofer, U. Nierste and D. Scherer, JHEP 0910 (2009) 081 [arXiv:0907.5408 [hep-ph]].

[29] M. Carena, D. Garcia, U. Nierste and C. Wagner, Nucl. Phys. B 577 (2000) 88 [arXiv:hep-ph/9912516].

[30] J. Guasch, P. Hafliger and M. Spira, Phys. Rev. D 68 (2003) 115001 [arXiv:hep-ph/0305101].

[31] D. Noth and M. Spira, Phys. Rev. Lett. 101 (2008) 181801 [arXiv:0808.0087 [hep-ph]]; JHEP 1106 (2011) 084 [arXiv:1001.1935 [hep-ph]].

[32] L. Mihaila and C. Reisser, JHEP 1008 (2010) 021 [arXiv:1007.0693 [hep-ph]].

[33] M. Carena, S. Heinemeyer, C. Wagner and G. Weiglein, Eur. Phys. J. C 26 (2003) 601 [arXiv:hep-ph/0202167].

[34] R. Barnett, H. Haber and D. Soper, Nucl. Phys. B 306 (1988) 697.

[35] R. Harlander, M. Krämer and M. Schumacher, arXiv:1112.3478 [hep-ph].

[36] F. Maltoni, G. Ridolfi and M. Ubiali, JHEP 1207 (2012) 022 [arXiv:1203.6393 [hep-ph]].

[37] R. Klees, M. Krämer and M. Ubiali, in preparation. 